\title{
Article \\ Effects of Vitamin D Supplementation on Surrogate Markers of Fertility in PCOS Women: A Randomized Controlled Trial
}

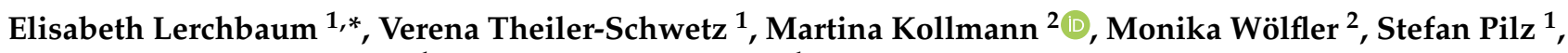 \\ Barbara Obermayer-Pietsch ${ }^{1}$ and Christian Trummer ${ }^{1}$ \\ 1 Department of Internal Medicine, Division of Endocrinology and Diabetology, Medical University of Graz, \\ Auenbruggerplatz 15, 8036 Graz, Austria; verena.schwetz@medunigraz.at (V.T.-S.); \\ stefan.pilz@medunigraz.at (S.P.); barbara.obermayer@medunigraz.at (B.O.-P.); \\ christian.trummer@medunigraz.at (C.T.) \\ 2 Department of Obstetrics and Gynecology, Division of Gynecological Endocrinology and Reproductive \\ Medicine, Medical University of Graz, Auenbruggerplatz 14, 8036 Graz, Austria; \\ martina.kollmann@medunigraz.at (M.K.); monika.woelfler@medunigraz.at (M.W.) \\ * Correspondence: elisabeth.lerchbaum@medunigraz.at; Tel.: +43-316-385-82383
}

check for updates

Citation: Lerchbaum, E.; Theiler-Schwetz, V.; Kollmann, M.; Wölfler, M.; Pilz, S.; Obermayer-Pietsch, B.; Trummer, C. Effects of Vitamin D Supplementation on Surrogate Markers of Fertility in PCOS Women: A Randomized Controlled Trial. Nutrients 2021, 13, 547. https:// doi.org/10.3390/nu13020547

Academic Editors: Luigi Barrea and Giovanna Muscogiuri

Received: 21 December 2020

Accepted: 2 February 2021

Published: 7 February 2021

Publisher's Note: MDPI stays neutral with regard to jurisdictional claims in published maps and institutional affiliations.

Copyright: (c) 2021 by the authors. Licensee MDPI, Basel, Switzerland. This article is an open access article distributed under the terms and conditions of the Creative Commons Attribution (CC BY) license (https:// creativecommons.org/licenses/by/ $4.0 /)$.

\begin{abstract}
Vitamin D (VD) might play an important role in polycystic ovary syndrome (PCOS) and female fertility. However, evidence from randomized controlled trials (RCT) is sparse. We examined VD effects on anti-Müllerian hormone (AMH) and other endocrine markers in PCOS and nonPCOS women. This is a post hoc analysis of a single-center, double-blind RCT conducted between December 2011 and October 2017 at the endocrine outpatient clinic at the Medical University of Graz, Austria. We included 180 PCOS women and 150 non-PCOS women with serum 25-hydroxyvitamin $\mathrm{D}(25(\mathrm{OH}) \mathrm{D})$ concentrations $<75 \mathrm{nmol} / \mathrm{L}$ in the trial. We randomized subjects to receive 20,000 IU of VD3/week (119 PCOS, 99 non-PCOS women) or placebo (61 PCOS, 51 non-PCOS women) for 24 weeks. Outcome measures were $\mathrm{AMH}$, follicle-stimulating hormone (FSH), luteinizing hormone (LH), estradiol, dehydroepiandrosterone sulfate, and androstenedione. In PCOS women, we observed a significant treatment effect on FSH (mean treatment effect $0.94,95 \%$ confidence interval [CI] 0.087 to $1.799, p=0.031$ ) and $\mathrm{LH} / \mathrm{FSH}$ ratio (mean treatment effect $-0.335,95 \% \mathrm{CI}-0.621$ to $0.050, p=0.022$ ), whereas no significant effect was observed in non-PCOS women. In PCOS women, VD treatment for 24 weeks had a significant effect on FSH and LH/FSH ratio but no effect on AMH levels.
\end{abstract}

Keywords: vitamin D; polycystic ovary syndrome; anti-Müllerian hormone; follicle-stimulating hormone; randomized controlled trial

\section{Introduction}

Vitamin D (VD) is a steroid hormone with well-known effects on calcium and bone metabolism [1]. Accumulating evidence from cross-sectional studies indicates an association of low 25-hydroxyvitamin D $(25(\mathrm{OH}) \mathrm{D})$ concentrations with various conditions including obesity, metabolic disorders [2,3], cardiovascular disease [4], hypogonadism [5], polycystic ovary syndrome (PCOS) [6], and decreased female fertility [7]. It has been hypothesized that a possible VD effect on ovarian anti-Müllerian hormone $(\mathrm{AMH}) \mathrm{might}$ be a putative component explaining the complex relationship of VD and human reproduction [8]. AMH is an ovarian biomarker playing a central role in folliculogenesis and ovarian dysfunction. Several in vitro as well as in vivo studies examined the potential effects of vitamin D on ovarian function $[9,10]$. Kinuta et al. [9] found that VD receptor null female mice suffer from ovarian insufficiency that is characterized by impaired follicular development. A recent meta-analysis assessed the reproductive outcomes of 2700 subfertile women and found a significant association of favorable outcomes with replete vitamin D status [10]. It has been hypothesized that VD acts upon the ovarian follicle and may improve oocyte quality [10]. As impaired ovarian function is also related to obesity, it should 
be mentioned that obesity is associated with low VD status due to decreased physical activity, low sun exposure, and sequestration in the adipose tissue [11,12]. Furthermore, it has been hypothesized that low $25(\mathrm{OH}) \mathrm{D}$ concentrations are involved in the development of obesity by influencing adipogenesis [12].

PCOS is the most common endocrine disorder among women of childbearing age [13]. Of note, PCOS has a very high prevalence and up to $10 \%$ of women of reproductive age are affected by PCOS [13]. In addition to hyperandrogenemia and metabolic disturbances such as obesity and insulin resistance, affected women frequently suffer from decreased fertility due to anovulation $[13,14]$. Moreover, alterations in lipid pattern are associated with obstetric complications in PCOS women [15]. Diet plays an important role in the pathogenesis of PCOS and obesity is related to the severity of the syndrome [16,17]. An increasing number of studies have examined the association of VD status with various features of PCOS. Whereas the majority of observational studies point towards a link of deficient VD status with obesity, metabolic disturbances, and anovulation, data derived from randomized controlled trials (RCTs) are limited [6]. Compared to healthy women, PCOS women have higher AMH levels and AMH is considered as an important diagnostic and prognostic marker in PCOS [18]. Existing cross-sectional studies on the association of 25(OH)D concentrations and AMH levels have reported inconsistent results [19]. Although a small RCT among VD-deficient infertile PCOS women reported a positive VD effect on AMH levels [20], data from large RCTs are lacking. Therefore, a recent systematic review and meta-analysis on VD and AMH concluded that large RCTs of VD supplementation are necessary to elucidate the complex relationship of VD and AMH [19].

Consequently, we performed a post hoc analysis of our RCT that was designed to examine VD effects on endocrine and metabolic parameters in PCOS and non-PCOS women. We aim to investigate VD effects on AMH levels as well as on other endocrine parameters involved in reproduction, including follicle-stimulating hormone (FSH), luteinizing hormone (LH), and estradiol in PCOS as well as in healthy premenopausal women without PCOS. Furthermore, we analyze VD effects on dehydroepiandrosterone sulfate (DHEAS) and androstenedione levels.

\section{Materials and Methods}

This study is a post hoc analysis of a single-center, placebo-controlled, double-blind, parallel-group study performed at the Medical University of Graz (MUG), Austria. We designed our study to examine VD effects on endocrine and metabolic parameters in PCOS as well as in healthy women without PCOS.

We have published details on the study design and methods previously $[21,22]$. The design, conducting, and publication of this study adhere to the recommendations of the CONSORT Statement (http:/ / www.consort-statement.org/). We registered the trial at http: / /www.clinicaltrialsregister.eu (EudraCT number, 2011-000994-30) and at clinicaltrials.gov (ClinicalTrials.gov Identifier NCT01721915). The local ethics committee approved the study protocol (EK 23-300 ex 10/11).

\subsection{Subjects}

Premenopausal women aged $\geq 18$ years with $25(\mathrm{OH}) \mathrm{D}$ concentrations $<75 \mathrm{nmol} / \mathrm{L}$ were eligible for our study. In the PCOS group, we established a diagnosis of PCOS using the Rotterdam criteria [23] if two out of the following three features were met: clinical and/or biochemical hyperandrogenism, polycystic ovaries, and/or oligo-/anovulation. We excluded disorders with similar clinical features before we made the diagnosis of PCOS. Non-PCOS women were required to show none of the Rotterdam PCOS criteria.

Exclusion criteria in both groups were hypercalcemia (defined as plasma calcium concentrations $>2.65 \mathrm{mmol} / \mathrm{L}$ ), regular vitamin D supplementation within 3 months prior to study inclusion, prevalent type 2 diabetes mellitus, use of insulin-sensitizing drugs (i.e., metformin, incretin mimetic drugs, thiazolidinedione, sulfonylurea) within 6 months prior to study inclusion, hormonal contraception within 3 months prior to study inclusion, use of 
lipid-lowering drugs or other drugs affecting insulin sensitivity or serum androgens (e.g., niacin, corticosteroids, beta-blockers, calcium channel blockers, thiazide diuretics) as well as disorders apart from PCOS associated with irregular menses and/or androgen excess.

We recruited PCOS and non-PCOS women from patients of the Division of Endocrinology and Diabetology and the Division of Gynecological Endocrinology and Reproductive Medicine at the MUG by conversation during routine visits in the outpatient clinics. Furthermore, we recruited participants from female hospital staff and female family members of hospital staff, and written information about the study was posted in the outpatient clinic. We informed all study participants during recruitment about the possibility of receiving a placebo.

\section{Healthy Women}

We included not only PCOS but also healthy women in our RCT as vitamin D might have varying effects among women with and without PCOS. As outlined above, the relationship of vitamin D and AMH is complex. Vitamin D might increase AMH levels in healthy women [24] but decrease AMH levels in PCOS women [20]. Therefore, to examine whether vitamin D effects vary depending on the respective group, we included PCOS as well as healthy women without PCOS in our analyses.

\subsection{Intervention}

We allocated subjects to the placebo (PBO) or VD group according to a computergenerated randomization list using a ratio of 2:1. We placed study medication into numbered bottles according to this list.

The VD group received an oral dose of 20,000 IU VD3 per week (equivalent to $2857 \mathrm{IU} /$ day) as 50 oily drops per week (Oleovit D3 drops; Fresenius Kabi Austria GmbH, Linz) for 24 weeks. Our PBO group received 50 oily drops without VD for 24 weeks. PBO oil contained the same oil as Oleovit D3 drops (without VD content). Fresenius Kabi Austria $\mathrm{GmbH}$, Linz delivered the PBO oil. All investigators involved in the enrollment of study subjects, data collection as well as assignment to intervention were masked to participant allocation. In order to improve and verify compliance, we asked study participants to return full as well as empty study medication bottles at the end of the study.

\subsection{Outcome Measures}

This is a post hoc analysis of our RCT including PCOS and non-PCOS women. We investigate VD effects on endocrine parameters including AMH, FSH, LH, estradiol, DHEAS, and androstenedione levels.

\subsection{Procedures}

We collected basal blood samples for measurement of 25(OH)D, AMH, FSH, LH, estradiol, DHEAS, and androstenedione between 8.00 and 9.00 a.m. after an overnight fast. We used 25(OH)D concentrations determined by immunoassay for evaluation of inclusion criteria. We performed biobanking of all remaining blood samples by freezing and storing at $-80{ }^{\circ} \mathrm{C}$ until analysis. In addition, we measured serum concentrations of $25(\mathrm{OH}) \mathrm{D}$ by well-adjusted isotope dilution-liquid chromatography tandem mass spectrometry (ID-LCMS/MS) methods in 2018 [21,22].

We measured FSH, LH, and estradiol levels on a daily basis. LH and FSH were measured using Access ${ }^{\circledR}$ hLH and hFSH CLIA (Beckman Coulter Inc., Brea, CA, USA), respectively. $17 \beta$-estradiol was determined using IMMULATE ${ }^{\circledR}$ CLIA assays (Siemens Healthcare Diagnostics Products Ltd., Glyn Rhonwy, UK). We measured AMH, androstenedione, and DHEAS levels once weekly, and blood samples were frozen and stored at $-40{ }^{\circ} \mathrm{C}$ until analysis. We measured DHEAS (Labor Diagnostika Nord, Nordhorn, Germany) and androstenedione (Siemens Healthcare Diagnostics Products Ltd.) via enzyme-linked immunosorbent assay (ELISA), with intra-assay and interassay coefficients of variation (CV) of $<10 \%$. In our laboratory, the assay for AMH was changed in November 2014 from the 
ultra-sensitive anti-Müllerian hormone/Müllerian-inhibiting substance enzyme-linked immunosorbent assay (ELISA) kit (Ansh Labs, Webster, TX, USA) to the Access 2 immunosorbent assay system (Beckmann Coulter). We compared both assays and found a good correlation $(\mathrm{r}=0.95)$. Both $\mathrm{AMH}$ assays show intra-assay and interassay $\mathrm{CV}$ of $<10 \%$. Laboratory kits and assays did not change between 2011 and 2017 for the remaining outcome parameters.

Vitamin D intake was assessed by questionnaires.

\subsection{Statistical Analyses}

We performed sample size calculation based on the data derived from a pilot study conducted among PCOS women [25]. In detail, we found a reduction area under the curve (AUC) glucose from $115 \pm 17$ at baseline to $103 \pm 18$ at the end of the study after 24 weeks VD supplementation. We therefore calculated a sample size of 92 participants to detect a treatment difference at two-sided 0.05 significance levels with a probability of $90 \%$, if the true difference between treatments is 12 with a standard deviation of 17 . As the analyses of VD effects according to genotype profile were a secondary outcome measure (results have been published previously [26]), we randomized study participants 2:1 (VD:PBO) in order to increase the sample size in the VD group. The number of enrolled PCOS subjects was increased from 150 to 180 to ensure an adequate power to detect differences regarding AUCgluc.

We used descriptive statistics as well as the Kolmogorov-Smirnov test to analyze the distribution of data. We present continuous data with normal distribution as means with SD and continuous data following a skewed distribution as median with interquartile range. We performed log transformation of skewed variables and rechecked log transformed data for normal distribution before parametric tests were performed. We used Student's T-test and $\chi^{2}$-test for comparisons of baseline characteristics between groups. Delta $(\Delta)$ values (value at the end of the study minus baseline value) were calculated for $25(\mathrm{OH}) \mathrm{D}$ and outcome measures. We used Pearson correlation analysis to determine relationships between variables. We performed multivariable stepwise linear regression analysis with $\mathrm{LH} / \mathrm{FSH}$ ratio and androstenedione as the dependent variables, and with BMI, age, and 25(OH)D as independent variables.

We executed analyses of outcome variables according to the intention-to-treat principle. In these analyses, we included all subjects with baseline and follow-up values. We applied analysis of covariance and adjusted our analyses for baseline values to test for differences in the respective outcome variables between the VD and the PBO group at the end of the study. We performed subgroup analyses of PCOS women with irregular menses. All statistical procedures were performed with SPSS version 26 (SPSS Inc., Chicago, IL, USA). We considered a $p$-value of $<0.05$ as statistically significant.

\section{Results}

We screened $\sim 500$ PCOS women and $~ 300$ healthy women without PCOS who routinely visited the endocrine outpatient clinic or responded to written information material for study eligibility. We randomized and enrolled 180 PCOS women and 150 healthy women in the study (participant flow charts have been published previously [21,22]).We randomized the first subject in December 2011 and we performed the last follow-up in October 2017.

In Table 1, we display the baseline characteristics of all study subjects. In PCOS women, we observed significantly higher BMI $(p=0.001)$, AMH levels $(p<0.001)$, LH levels $(p=0.02)$, LH/FSH ratio $(p<0.001)$, DHEAS $(p<0.001)$, and androstenedione levels $(p<0.001)$, whereas age $(p<0.001), 25(\mathrm{OH}) \mathrm{D}(p=0.019), \mathrm{FSH}(p<0.001)$, and estradiol levels $(p<0.001)$ were lower compared to healthy women without PCOS. PCOS women in the VD group were significantly younger compared to PCOS women in the PBO group. In healthy women without PCOS, baseline estradiol levels were significantly lower in the VD group compared to the PBO group. We found no significant differences among the 
remaining baseline characteristics between VD and PBO groups in PCOS as well as in healthy women.

Table 1. Baseline characteristics of study subjects. Data are shown as means with standard deviation, median, and interquartile range or proportion as appropriate. PCOS—polycystic ovary syndrome; VD—vitamin D; PBO—placebo; BMI-body mass index; 25(OH)D—25-hydroxyvitamin D; AMH—anti-Müllerian hormone; FSH—follicle-stimulating hormone; LH_luteinizing hormone; DHEAS—dehydroepiandrosterone sulfate. We performed comparisons of baseline characteristics between women in the VD and the PBO groups using Student's $t$-test and $\chi^{2}$-test. Season 1: January-March; season 2: April-June; season 3: July-September; season 4: October-December.

\begin{tabular}{|c|c|c|c|c|}
\hline & $\begin{array}{l}\text { All PCOS Women } \\
\quad(n=180)\end{array}$ & $\operatorname{VD}(n=119)$ & PBO $(n=61)$ & $p$-Value \\
\hline Age (years) & $26.0 \pm 5.0$ & $25.4 \pm 4.6$ & $27.2 \pm 5.5$ & 0.022 \\
\hline $\mathrm{BMI}\left(\mathrm{kg} / \mathrm{m}^{2}\right)$ & $27.6 \pm 7.5$ & $27.3 \pm 7.4$ & $28.3 \pm 7.8$ & 0.453 \\
\hline $25(\mathrm{OH}) \mathrm{D} *(\mathrm{nmol} / \mathrm{L})$ & $50.4 \pm 19.0$ & $50.7 \pm 19.5$ & $49.9 \pm 18.3$ & 0.798 \\
\hline $\mathrm{AMH}(\mathrm{ng} / \mathrm{mL})$ & $7.67(4.09-15.0)$ & $7.62(4.23-15.0)$ & $7.71(3.15-15.0)$ & 0.547 \\
\hline $\mathrm{FSH}(\mu \mathrm{U} / \mathrm{mL})$ & $5.97 \pm 2.41$ & $6.04 \pm 2.59$ & $5.94 \pm 2.33$ & 0.783 \\
\hline $\mathrm{LH}(\mu \mathrm{U} / \mathrm{mL})$ & $8.88(4.26-14.5)$ & $8.89(4.20-15.34)$ & $8.86(3.82-14.18)$ & 0.830 \\
\hline $\mathrm{LH} / \mathrm{FSH}$ ratio & $1.48(0.88-2.30)$ & $1.52(0.88-2.54)$ & $1.38(0.68-2.55)$ & 0.530 \\
\hline Estradiol (pg/mL) & $60.6(41.0-122.0)$ & $59.1(39.3-123.0)$ & $64.0(43.5-158.0)$ & 0.311 \\
\hline DHEAS $(\mu \mathrm{g} / \mathrm{mL})$ & $1.90(1.24-2.97)$ & $1.94(1.16-3.22)$ & $1.9(1.28-3.07)$ & 0.789 \\
\hline Androstenedione $(\mathrm{ng} / \mathrm{mL})$ & $3.36(2.26-4.87)$ & $2.4(1.48-4.24)$ & $2.61(1.79-3.96)$ & 0.937 \\
\hline Vitamin D intake (IU/day) & $31(14-76)$ & $31(16-67)$ & $31(13-78)$ & 0.582 \\
\hline \multicolumn{5}{|l|}{ Season of recruitment } \\
\hline Season 1 & $38.3 \%$ & $36.1 \%$ & $42.6 \%$ & 0.442 \\
\hline Season 2 & $26.1 \%$ & $26.1 \%$ & $26.2 \%$ & \\
\hline Season 3 & $17.8 \%$ & $21.0 \%$ & $11.5 \%$ & \\
\hline \multirow[t]{2}{*}{ Season 4} & $17.8 \%$ & $16.8 \%$ & $19.7 \%$ & \\
\hline & $\begin{array}{l}\text { All Healthy Women } \\
(n=150)\end{array}$ & VD $(n=99)$ & PBO $(n=51)$ & \\
\hline Age (years) & $35.8 \pm 8.7$ & $35.7 \pm 8.9$ & $36.1 \pm 8.4$ & 0.826 \\
\hline $\mathrm{BMI}\left(\mathrm{kg} / \mathrm{m}^{2}\right)$ & $25.2 \pm 5.5$ & $25.5 \pm 5.3$ & $24.7 \pm 5.8$ & 0.398 \\
\hline $25(\mathrm{OH}) \mathrm{D} *(\mathrm{nmol} / \mathrm{L})$ & $55.4 \pm 18.9$ & $55.4 \pm 18.9$ & $55.3 \pm 18.9$ & 0.996 \\
\hline $\mathrm{AMH}(\mathrm{ng} / \mathrm{mL})$ & $1.97(0.32-4.38)$ & $1.89(0.29-5.2)$ & $2.41(0.32-5.30)$ & 0.546 \\
\hline $\mathrm{FSH}(\mu \mathrm{U} / \mathrm{mL})$ & $9.86 \pm 13.11$ & $9.67 \pm 12.05$ & $9.96 \pm 13.69$ & 0.898 \\
\hline $\mathrm{LH}(\mu \mathrm{U} / \mathrm{mL})$ & $6.28(3.72-11.0)$ & $6.28(3.24-11.50)$ & $6.48(4.04-14.20)$ & 0.119 \\
\hline LH/FSH ratio & $0.93(0.51-1.59)$ & $0.87(0.48-1.57)$ & $1.12(10.51-2.03)$ & 0.242 \\
\hline Estradiol (pg/mL) & $92.6(50.5-156.0)$ & $83.4(41.5-145)$ & $114(61.1-212.0)$ & 0.006 \\
\hline DHEAS $(\mu \mathrm{g} / \mathrm{mL})$ & $1.21(0.78-2.0)$ & $1.20(0.75-2.03)$ & $1.23(0.76-2.19)$ & 0.508 \\
\hline Androstenedione (ng/mL) & $2.50(1.56-3.96)$ & $2.4(1.48-4.24)$ & $2.61(1.79-3.96)$ & 0.642 \\
\hline Vitamin D intake (IU/day) & $50(26-77)$ & $50(22-80)$ & $50(27-72)$ & 0.471 \\
\hline \multicolumn{5}{|l|}{ Season of recruitment } \\
\hline Season 1 & $30.7 \%$ & $29.3 \%$ & $33.3 \%$ & 0.942 \\
\hline Season 2 & $32.7 \%$ & $32.3 \%$ & $33.3 \%$ & \\
\hline Season 3 & $10.0 \%$ & $10.1 \%$ & $9.8 \%$ & \\
\hline Season 4 & $26.7 \%$ & $28.3 \%$ & $23.5 \%$ & \\
\hline
\end{tabular}

\footnotetext{
${ }^{*}$ We measured 25(OH)D by liquid chromatography tandem mass spectrometry.
} 


\subsection{Cross-Sectional Analyses}

In PCOS women, we found a significant correlation of 25(OH)D levels with $\mathrm{LH} / \mathrm{FSH}$ ratio $(r=-0.195, p=0.009)$ as well as with androstenedione levels $(r=0.15, p=0.043)$. We observed no significant correlation of $25(\mathrm{OH}) \mathrm{D}$ levels with the remaining endocrine parameters (AMH, LH, FSH, estradiol, and DHEAS). In analyses adjusted for age and $\mathrm{BMI}$, the correlation of $25(\mathrm{OH}) \mathrm{D}$ with $\mathrm{LH} / \mathrm{FSH}$ ratio $(p=0.011)$ remained stable but was attenuated for androstenedione $(p=0.070)$.

In healthy women, we observed no significant correlation of $25(\mathrm{OH}) \mathrm{D}$ with endocrine parameters.

\subsection{Outcome Analyses}

\subsubsection{PCOS Women}

In PCOS women, the mean $( \pm \mathrm{SD})$ overall treatment period was $176 \pm 23$ days in the VD group and $176 \pm 21$ days in the PBO group $(p=0.906)$. A total of 123 study participants completed both study visits.

In Table 2, we display results of outcome analyses. In PCOS women, we found a significant VD effect on FSH levels as well as on LH/FSH ratio. We found no significant effect on the remaining outcome parameters. After exclusion of PCOS women with regular menses $(n=19)$, VD effects on FSH levels (mean treatment effect $0.271,95 \%$ CI 0.27 to $2.06, p=0.011$ ) and $\mathrm{LH} / \mathrm{FSH}$ ratio (mean treatment effect $-0.401,95 \% \mathrm{CI}-0.705$ to -0.097 , $p=0.010)$ remained stable.

Table 2. Continuous outcome variables at baseline and end of the study in PCOS women with available values at both study visits. We display data as means with standard deviation or medians and interquartile range as appropriate. We calculated treatment effects with 95\% confidence interval and $p$-values by analysis of covariance for group differences at the end of the study. Analyses were adjusted for baseline values. IQR -interquartile range; AMH—anti-Müllerian hormone; VD—vitamin D; PBO_placebo; FSH—follicle-stimulating hormone; LH—luteinizing hormone; DHEAS—dehydroepiandrosterone sulfate.

\begin{tabular}{|c|c|c|c|c|}
\hline & Baseline Visit & Study End & $\begin{array}{l}\text { Treatment Effect }(95 \% \\
\text { Confidence Interval) }\end{array}$ & $p$-Value \\
\hline \multicolumn{5}{|c|}{$\mathrm{AMH}^{*}(\mathrm{ng} / \mathrm{mL})$} \\
\hline $\mathrm{VD}(n=80)$ & $7.6(4.2-15.0)$ & $7.0(4.2-15.5)$ & \multirow{2}{*}{$0.097(-0.081$ to 0.276$)$} & \multirow{2}{*}{0.282} \\
\hline $\mathrm{PBO}(n=40)$ & $7.7(3.2-15.0)$ & $7.6(2.8-14.4)$ & & \\
\hline \multicolumn{5}{|c|}{$\mathrm{FSH}(\mu \mathrm{U} / \mathrm{mL})$} \\
\hline $\operatorname{VD}(n=81)$ & $6.04 \pm 2.59$ & $6.16 \pm 2.46$ & \multirow{2}{*}{0.94 (0.087 to 1.799$)$} & \multirow{2}{*}{0.031} \\
\hline $\mathrm{PBO}(n=41)$ & $5.94 \pm 2.33$ & $5.23 \pm 1.78$ & & \\
\hline \multicolumn{5}{|c|}{$\mathrm{LH}^{*}(\mu \mathrm{U} / \mathrm{mL})$} \\
\hline $\operatorname{VD}(n=79)$ & $8.9(4.2-15.3)$ & $9.4(3.4-15.2)$ & \multirow{2}{*}{$-0.184(-0.497$ to 0.129$)$} & \multirow{2}{*}{0.248} \\
\hline $\mathrm{PBO}(n=41)$ & $8.9(3.8-14.2)$ & $8.8(4.1-14.7)$ & & \\
\hline \multicolumn{5}{|c|}{ Estradiol * $(\mathrm{pg} / \mathrm{mL})$} \\
\hline $\operatorname{VD}(n=81)$ & $59.1(39.3-123.0)$ & $59.4(33.9-169.0)$ & \multirow{2}{*}{$-0.096(-0.351$ to 0.159$)$} & \multirow{2}{*}{0.460} \\
\hline $\mathrm{PBO}(n=41)$ & $64.0(43.5-158.0)$ & $73.8(44.2-193.0)$ & & \\
\hline \multicolumn{5}{|c|}{ LH/FSH ratio * } \\
\hline $\operatorname{VD}(n=79)$ & $1.52(0.88-2.54)$ & $1.45(0.79-2.73)$ & \multirow{2}{*}{$-0.335(-0.621$ to -0.050$)$} & \multirow{2}{*}{0.022} \\
\hline $\mathrm{PBO}(n=41)$ & $1.38(0.69-2.55)$ & $1.73(0.76-3.32)$ & & \\
\hline & & DHEAS * $(\mu \mathrm{g} / \mathrm{mL})$ & & \\
\hline
\end{tabular}


Table 2. Cont.

\begin{tabular}{|c|c|c|c|c|}
\hline & Baseline Visit & Study End & $\begin{array}{l}\text { Treatment Effect (95\% } \\
\text { Confidence Interval) }\end{array}$ & $p$-Value \\
\hline $\operatorname{VD}(n=81)$ & $1.94(1.16-3.22)$ & $1.96(1.06-3.12)$ & \multirow{2}{*}{$-0.016(-0.142$ to 0.11$)$} & \multirow{2}{*}{0.805} \\
\hline $\mathrm{PBO}(n=41)$ & $1.9(1.28-3.07)$ & $2.12(1.31-3.23)$ & & \\
\hline \multicolumn{5}{|c|}{ Androstenedione * $(\mathrm{ng} / \mathrm{mL})$} \\
\hline $\operatorname{VD}(n=80)$ & $3.41(2.24-4.95)$ & $3.68(2.55-6.0)$ & \multirow{2}{*}{$0(-0.131$ to 0.130$)$} & \multirow{2}{*}{0.996} \\
\hline $\operatorname{PBO}(n=40)$ & $3.32(2.05-5.58)$ & $3.86(2.33-7.11)$ & & \\
\hline
\end{tabular}

* Skewed variables for which logarithmic transformed values were used in ANCOVA, but untransformed values are shown in the table.

We observed a significant negative correlation of $\Delta 25(\mathrm{OH}) \mathrm{D}$ levels with $\triangle \mathrm{LH} / \mathrm{FSH}$ ratio $(r=-0.208, p=0.024)$ and a trend with $\Delta \mathrm{FSH}(r=0.169, p=0.066)$. We observed no significant correlation of $\Delta 25(\mathrm{OH}) \mathrm{D}$ with $\triangle \mathrm{AMH}, \Delta \mathrm{LH}, \Delta$ estradiol, $\Delta$ androstenedione, and $\triangle$ DHEAS $(p>0.05$ for all).

Table 3 shows $25(\mathrm{OH}) \mathrm{D}$ concentrations at baseline and the end of the study in PCOS women. VD supplementation significantly increased 25(OH)D concentrations.

Table 3. 25(OH)D concentrations at baseline and at the end of the study in subjects with available values at both study visits. Data are shown as means with standard deviation. Treatment effects with $95 \%$ confidence interval and $p$-values were calculated by ANCOVA for group differences at follow-up with adjustment for baseline value.

\begin{tabular}{|c|c|c|c|c|}
\hline & Baseline & Follow-Up (24 Weeks) & Treatment Effect (95\% Confidence Interval) & $p$-Value \\
\hline \multicolumn{5}{|c|}{ PCOS women } \\
\hline \multicolumn{5}{|c|}{$25(\mathrm{OH}) \mathrm{D}(\mathrm{nmol} / \mathrm{L})$} \\
\hline $\operatorname{VD}(n=79)$ & $48.8 \pm 16.8$ & $90.2 \pm 20.1$ & \multirow{2}{*}{33.4 (24.5 to 42.2$)$} & \multirow{2}{*}{$<0.001$} \\
\hline $\mathrm{PBO}(n=44)$ & $48.8 \pm 17.5$ & $56.8 \pm 29.5$ & & \\
\hline \multicolumn{5}{|c|}{ Healthy women } \\
\hline \multicolumn{5}{|c|}{$25(\mathrm{OH}) \mathrm{D}(\mathrm{nmol} / \mathrm{L})$} \\
\hline $\operatorname{VD}(n=82)$ & $55.8 \pm 19.9$ & $95.3 \pm 26.2$ & \multirow{2}{*}{28.5 (19.3 to 37.7$)$} & \multirow{2}{*}{$<0.001$} \\
\hline $\mathrm{PBO}(n=44)$ & $56.2 \pm 19.3$ & $67.0 \pm 24.8$ & & \\
\hline
\end{tabular}

PCOS—polycystic ovary syndrome; 25(OH)D—25-hydroxyvitamin D; VD—vitamin D; PBO—placebo.

VD effects on metabolic parameters are shown in supplemental Tables S1 and S2. In PCOS women, we found a significant beneficial VD effect on glucose levels at $60 \mathrm{~min}$ during the oral glucose tolerance test (Supplementary Table S1). In non-PCOS women, VD treatment had a significant unfavorable effect on insulin resistance and insulin sensitivity (Supplementary Table S2).

\subsubsection{Non-PCOS Women}

In healthy women, the mean $( \pm \mathrm{SD})$ treatment duration was $174 \pm 44$ days in the VD group and $173 \pm 23$ days in the PBO group ( $p=0.884$ ). In total, 127 participants completed the entire study including the last follow-up visit after 24 weeks.

In healthy women without PCOS, we found no significant VD effect on outcome measures $(p>0.05$ for all, data not shown). Furthermore, we observed no significant correlation of $\Delta 25(\mathrm{OH}) \mathrm{D}$ with changes in outcome measures ( $p>0.05$ for all).

Table 3 shows 25(OH)D concentrations at baseline and the end of the study in healthy women. We found a significant VD effect on $25(\mathrm{OH}) \mathrm{D}$ concentrations in women without PCOS. 


\section{Discussion}

In our RCT in PCOS women with baseline $25(\mathrm{OH}) \mathrm{D}$ concentrations $<75 \mathrm{nmol} / \mathrm{L}, \mathrm{VD}$ treatment had a significant effect on FSH levels and LH/FSH ratio. We found, however, no significant VD effect on AMH levels and the remaining endocrine parameters. In healthy women with serum $25(\mathrm{OH}) \mathrm{D}<75 \mathrm{nmol} / \mathrm{L}$ at baseline, we observed no significant VD effect on outcome measures.

Interestingly, we observed a significant VD effect on FSH levels and LH/FSH ratio as well as a significant correlation between $\Delta 25(\mathrm{OH}) \mathrm{D}$ and $\triangle \mathrm{LH} / \mathrm{FSH}$ ratio in PCOS women. In the pathophysiology of PCOS, abnormalities of the hypothalamic-pituitaryovarian axis play an important role [13]. A relative increase in LH to FSH release is caused by a disturbance in the secretion pattern of the gonadotrophin-releasing hormone [27]. Furthermore, ovarian estrogen is responsible for causing an abnormal feedback mechanism that results in increased LH release [28]. An elevated LH/FSH ratio is a common finding in PCOS and as a result, ovulation does not occur in many PCOS patients [29]. It has been reported that VD alters FSH sensitivity, indicating a possible physiological role for VD in the development and luteinization of the ovarian follicle [8]. Among induced PCOS rats, VD treatment increases the normal follicle number through increasing FSH and estradiol and decreasing LH [30]. Furthermore, Kinuta et al. [9] demonstrated that VD promoted folliculogenesis and follicular development in PCOS rats by increasing progesterone and estrogen levels and regulating the $\mathrm{LH} / \mathrm{FSH}$ ratio.

Our results contribute to the mounting evidence from cross-sectional and interventional studies on favorable VD effects on reproduction [7,31,32]. It has been hypothesized that physiological levels of VD might have a beneficial role in ovulation and endometrial receptivity [33]. Consistently, findings from systematic reviews and meta-analyses analyzing the association of VD and assisted reproduction outcomes suggest that women with replete VD status have more live births, more positive pregnancy tests, and more clinical pregnancies compared to women with deficient or insufficient $25(\mathrm{OH}) \mathrm{D}[31,32]$. Recently, Butts et al. [31] reported that VD deficiency in PCOS women who underwent ovarian stimulation for infertility treatment was linked with significantly diminished rates of ovulation, of pregnancy, and ultimately, a reduced chance of live birth. Of note, there was no significant association of VD deficiency with ovulation, pregnancy, or live birth in non-PCOS women with unexplained infertility [31]. In light of the high prevalence of insufficient VD levels in PCOS women [6] and the significant burden of decreased fertility in affected women, our findings deserve investigation in future large RCTs including PCOS women as well as women without PCOS. Considering the fact that VD supplementation is a safe and cheap treatment, our findings might be of high clinical interest. It should, however, be emphasized that the clinical relevance of our findings regarding reproduction remains to be determined as we investigated only surrogate parameters involved in fertility.

We failed to find a significant VD effect on AMH levels. Existing evidence on the relationship of VD and AMH levels is conflicting [19]. It has been shown that VD regulates AMH levels in vitro, both directly through the AMH promoter [34] and indirectly by regulating the number of granulosa cells and AMH signaling in cultures of ovarian follicles [35]. In contrast to the consistency of the in vitro data, the evidence of a link between VD and AMH in women is contentious. The majority of cross-sectional studies failed to find a significant correlation of 25(OH)D levels and AMH [19]. In contrast, a prospective study including PCOS women observed an association of VD supplementation with a decrease in serum AMH levels [36]. Furthermore, positive VD effects on AMH levels were found in a prospective study including infertile women with diminished ovarian reserve [24]. To date, there are only two small RCTs investigating VD effects on AMH levels in women [20,37]. In a study among VD-deficient infertile PCOS women, participants received either 50,000 IU VD/week $(n=17)$ or PBO $(n=17)$ for 8 weeks. The authors found a significant decrease in AMH levels in the VD group compared with PBO [20]. Dennis et al. [37] conducted an RCT in 49 young women with regular menses to evaluate the effects of a single high dose of VD (50,000 IU, taken on the first day of the menstrual cycle) versus PBO on AMH levels during 
the following week. Interestingly, the authors observed a significant progressive increase in AMH levels in the following week after VD supplementation. In our RCT, we found no significant VD effect on AMH levels in PCOS or non-PCOS women. These different results might be related to varying VD doses, study duration, baseline $25(\mathrm{OH}) \mathrm{D}$ levels, age, and sample size. However, as $25(\mathrm{OH}) \mathrm{D}$ concentrations at the end of the study were high in both groups, it is unlikely that the lack of a significant VD effect on AMH levels in our study is related to insufficient vitamin D doses.

Our study has several limitations. First, as we investigated women with relatively high baseline 25(OH)D levels, we cannot exclude significant VD effects on AMH levels in women with lower baseline $25(\mathrm{OH}) \mathrm{D}$ levels. Another possible limitation is the relatively high drop-out rate in PCOS women. Furthermore, since blood samples were collected regardless of the participants' menstrual cycle, the results regarding some of the measured parameters (e.g., FSH, LH, estradiol) should be interpreted with caution. As gonadotropins vary consistently during the phases of the menstrual cycle, our results regarding vitamin D effects on FSH and LH/FSH ratio should be interpreted in light of this limitation. We cannot rule out that blood sampling in the first week of the menstrual cycle in PCOS women with a regular menstrual cycle would provide different results. Nevertheless, only a small number of PCOS women had a regular menstrual cycle $(n=19)$ and the exclusion of these PCOS women from our analyses did not materially change our results. Moreover, AMH levels are stable across the menstrual cycle and typically demonstrate minimal intercycle and intracycle variability [38,39]. As we did not assess data on sun exposure, we were not able to adjust our analyses for this potential confounder. Furthermore, we did not assess the dietary pattern of study participants. This limitation might influence our results, as it has been demonstrated that specific diets such as the Mediterranean diet are associated with circulating 25(OH)D concentrations [40]. Finally, our findings should be interpreted with caution because our results derive from a post hoc analysis and we did not adjust for multiple testing as our analyses were all based on a priori pre-specified hypotheses. Nevertheless, we cannot exclude that our statistical analyses revealed a false-positive finding. The strengths of our study include its design as an RCT, the large sample size as well as the inclusion of PCOS and non-PCOS women.

\section{Conclusions}

In summary, we found no significant VD effect on AMH levels but a significant effect on FSH levels and LH/FSH ratio in PCOS women. Our results, therefore, support the idea that VD may be involved in reproductive function in PCOS women. In light of previous data suggesting a possible favorable VD effect on female fertility, further adequately powered RCTs are of clinical importance to clarify the potential positive effects of VD on reproductive function in PCOS women.

Supplementary Materials: The following are available online at https:/ / www.mdpi.com/2072-664 3/13/2/547/s1, Table S1: Outcome variables at baseline and follow-up at study end in PCOS women with available values at both study visits, Table S2: Outcome variables at baseline and follow-up at study end in non-PCOS women with available values at both study visits.

Author Contributions: Conceptualization, E.L., B.O.-P.; methodology, E.L., S.P.; formal analysis, E.L.; investigation, M.K., M.W., B.O.-P.; resources, M.K., M.W., B.O.-P.; data curation, E.L., C.T., V.T.-S.; writing—original draft preparation, E.L.; writing—review and editing, S.P., C.T., V.T.-S.; supervision, B.O.-P.; project administration, E.L., V.T.-S., B.O.-P.; funding acquisition, E.L. All authors have read and agreed to the published version of the manuscript.

Funding: This study was supported by funding from the Austrian Science Fund (FWF), project no.: KLI 274.

Informed Consent Statement: Informed consent was obtained from all subjects involved in the study.

Data Availability Statement: The data presented in this study are available on request from the corresponding author. 
Acknowledgments: First, we thank all study subjects for participating in our study. Roswitha Gumpold recruited study participants. Furthermore, the Endocrinology Lab platform and Cornelia Missbrenner continuously supported our study and Fresenius Kabi provided the study medication. Open Access Funding by the Austrian Science Fund (FWF).

Conflicts of Interest: The authors declare no conflict of interest.

\section{References}

1. Holick, M.F. Vitamin D deficiency. N. Engl. J. Med. 2007, 357, 266-281. [CrossRef]

2. Wehr, E.; Pilz, S.; Schweighofer, N.; Giuliani, A.; Kopera, D.; Pieber, T.R.; Obermayer-Pietsch, B. Association of hypovitaminosis D with metabolic disturbances in polycystic ovary syndrome. Eur. J. Endocrinol. 2009, 161, 575-582. [CrossRef]

3. Trummer, C.; Pilz, S.; Schwetz, V.; Obermayer-Pietsch, B.; Lerchbaum, E. Vitamin D, PCOS and androgens in men: A systematic review. Endocr. Connect. 2018, 7, R95-R113. [CrossRef]

4. Pilz, S.; März, W.; Wellnitz, B.; Seelhorst, U.; Fahrleitner-Pammer, A.; Dimai, H.P.; Boehm, B.O.; Dobnig, H. Association of vitamin D deficiency with heart failure and sudden cardiac death in a large cross-sectional study of patients referred for coronary angiography. J. Clin. Endocrinol. Metab. 2008, 93, 3927-3935. [CrossRef]

5. Lerchbaum, E.; Pilz, S.; Trummer, C.; Rabe, T.; Schenk, M.; Heijboer, A.C.; Obermayer-Pietsch, B. Serum vitamin D levels and hypogonadism in men. Andrology 2014, 2, 748-754. [CrossRef]

6. $\mathrm{Mu}$, Y.; Cheng, D.; Yin, T.L.; Yang, J. Vitamin D and polycystic ovary syndrome: A narrative review. Reprod. Sci. 2020. [CrossRef]

7. Lerchbaum, E.; Rabe, T. Vitamin D and female fertility. Curr. Opin. Obstet. Gynecol. 2014, 26, 145-150. [CrossRef]

8. Irani, M.; Merhi, Z. Role of vitamin D in ovarian physiology and its implication in reproduction: A systematic review. Fertil. Steril. 2014, 102, 460-468. [CrossRef]

9. Kinuta, K.; Tanaka, H.; Moriwake, T.; Aya, K.; Kato, S.; Seino, Y. Vitamin D is an important factor in estrogen biosynthesis of both female and male gonads. Endocrinology 2000, 141, 1317-1324. [CrossRef]

10. Chu, J.; Gallos, I.; Tobias, A.; Tan, B.; Eapen, A.; Coomarasamy, A. Vitamin D and assisted reproductive treatment outcome: A systematic review and meta-analysis. Hum. Reprod. 2018, 33, 65-80. [CrossRef]

11. Savastano, S.; Barrea, L.; Savanelli, M.C.; Nappi, F.; Di Somma, C.; Orio, F.; Colao, A. Low vitamin D status and obesity: Role of nutritionist. Rev. Endocr. Metab. Disord. 2017, 18, 215-225. [CrossRef] [PubMed]

12. Barrea, L.; Frias-Toral, E.; Pugliese, G.; Garcia-Velasquez, E.; De Los Angeles Carignano, M.; Savastano, S.; Colao, A.; Muscogiuri, G. Vitamin D in obesity and obesity-related diseases: An overview. Minerva Endocrinol. 2020. [CrossRef]

13. Goodarzi, M.O.; Dumesic, D.A.; Chazenbalk, G.; Azziz, R. Polycystic ovary syndrome: Etiology, pathogenesis and diagnosis. Nat Rev. Endocrinol. 2011, 7, 219-231. [CrossRef]

14. Norman, R.J.; Dewailly, D.; Legro, R.S.; Hickey, T.E. Polycystic ovary syndrome. Lancet 2007, 370, 685-697. [CrossRef]

15. Palomba, S.; Falbo, A.; Chiossi, G.; Muscogiuri, G.; Fornaciari, E.; Orio, F.; Tolino, A.; Colao, A.; La Sala, G.B.; Zullo, F. Lipid profile in nonobese pregnant women with polycystic ovary syndrome: A prospective controlled clinical study. Steroids 2014, 88 , 36-43. [CrossRef]

16. Barrea, L.; Arnone, A.; Annunziata, G.; Muscogiuri, G.; Laudisio, D.; Salzano, C.; Pugliese, G.; Colao, A.; Savastano, S. Adherence to the Mediterranean diet, dietary patterns and body composition in women with Polycystic Ovary Syndrome (PCOS). Nutrients 2019, 11, 2278. [CrossRef]

17. Barrea, L.; Marzullo, P.; Muscogiuri, G.; Di Somma, C.; Scacchi, M.; Orio, F.; Aimaretti, G.; Colao, A.; Savastano, S. Source and amount of carbohydrate in the diet and inflammation in women with polycystic ovary syndrome. Nutr. Res. Rev. 2018, 31, 291-301. [CrossRef]

18. Qi, X.; Pang, Y.; Qiao, J. The role of anti-Müllerian hormone in the pathogenesis and pathophysiological characteristics of polycystic ovary syndrome. Eur. J. Obstet. Gynecol. Reprod. Biol. 2016, 199, 82-87. [CrossRef]

19. Moridi, I.; Chen, A.; Tal, O.; Tal, R. The Association between Vitamin D and Anti-Müllerian Hormone: A systematic review and meta-analysis. Nutrients 2020, 12, 1567. [CrossRef] [PubMed]

20. Dastorani, M.; Aghadavod, E.; Mirhosseini, N.; Foroozanfard, F.; Zadeh Modarres, S.; Amiri Siavashani, M.; Asemi, Z. The effects of vitamin D supplementation on metabolic profiles and gene expression of insulin and lipid metabolism in infertile polycystic ovary syndrome candidates for in vitro fertilization. Reprod. Biol. Endocrinol. 2018, 16, 94. [CrossRef]

21. Trummer, C.; Schwetz, V.; Kollmann, M.; Wölfler, M.; Münzker, J.; Pieber, T.R.; Pilz, S.; Heijboer, A.C.; Obermayer-Pietsch, B.; Lerchbaum, E. Effects of vitamin D supplementation on metabolic and endocrine parameters in PCOS: A randomized-controlled trial. Eur. J. Nutr. 2019, 58, 2019-2028. [CrossRef]

22. Trummer, C.; Theiler-Schwetz, V.; Kollmann, M.; Wölfler, M.; Münzker, J.; Pilz, S.; Pieber, T.R.; Heijboer, A.C.; Obermayer-Pietsch B.; Lerchbaum, E. Effects of vitamin D supplementation on metabolic and endocrine parameters in healthy premenopausal women: A randomized controlled trial. Clin. Nutr. 2020, 39, 718-726. [CrossRef]

23. Rotterdam ESHRE/ASRM-Sponsored PCOS Consensus Workshop Group. Revised 2003 consensus on diagnostic criteria and long-term health risks related to polycystic ovary syndrome (PCOS). Hum. Reprod. 2004, 19, 41-47. [CrossRef]

24. Naderi, Z.; Kashanian, M.; Chenari, L.; Sheikhansari, N. Evaluating the effects of administration of 25-hydroxyvitamin D supplement on serum anti-mullerian hormone (AMH) levels in infertile women. Gynecol. Endocrinol. 2018, 34, 409-412. [CrossRef] [PubMed] 
25. Wehr, E.; Pieber, T.R.; Obermayer-Pietsch, B. Effect of vitamin D3 treatment on glucose metabolism and menstrual frequency in polycystic ovary syndrome women: A pilot study. J. Endocrinol. Investig. 2011, 34, 757-763. [PubMed]

26. Trummer, O.; Schweighofer, N.; Haudum, C.W.; Trummer, C.; Pilz, S.; Theiler-Schwetz, V.; Keppel, M.H.; Grübler, M.; Pieber, T.R.; Renner, W.; et al. Genetic components of 25-Hydroxyvitamin D increase in three randomized controlled trials. J. Clin. Med. 2020, 9, 570. [CrossRef]

27. Yen, S.S. The polycystic ovary syndrome. Clin. Endocrinol. 1980, 12, 177-207. [CrossRef]

28. McKenna, T.J. Pathogenesis and treatment of polycystic ovary syndrome. N. Engl. J. Med. 1988, 318, 558-562. [CrossRef]

29. Johansson, J.; Stener-Victorin, E. Polycystic ovary syndrome: Effect and mechanisms of acupuncture for ovulation induction. Evid. Based Complement. Alternat. Med. 2013, 2013, 762615. [CrossRef]

30. Behmanesh, N.; Abedelahi, A.; Charoudeh, H.N.; Alihemmati, A. Effects of vitamin D supplementation on follicular development, gonadotropins and sex hormone concentrations, and insulin resistance in induced polycystic ovary syndrome. Turk. J. Obstet. Gynecol. 2019, 16, 143-150. [CrossRef] [PubMed]

31. Butts, S.F.; Seifer, D.B.; Koelper, N.; Senapati, S.; Sammel, M.D.; Hoofnagle, A.N.; Kelly, A.; Krawetz, S.A.; Santoro, N.; Zhang, H.; et al. Eunice kennedy shriver national institute of child health and human development reproductive medicine network. Vitamin D deficiency is associated with poor ovarian stimulation outcome in PCOS but not unexplained infertility. J. Clin. Endocrinol. Metab. 2019, 104, 369-378. [CrossRef]

32. Pilz, S.; Zittermann, A.; Obeid, R.; Hahn, A.; Pludowski, P.; Trummer, C.; Lerchbaum, E.; Pérez-López, F.R.; Karras, S.N.; März, W. The role of Vitamin D in fertility and during pregnancy and lactation: A review of clinical data. Int. J. Environ. Res. Public Health 2018, 15, 2241. [CrossRef]

33. Firouzabadi, R.D.; Rahmani, E.; Rahsepar, M.; Firouzabadi, M.M. Value of follicular fluid vitamin D in predicting the pregnancy rate in an IVF program. Arch. Gynecol. Obstet. 2014, 289, 201-206. [CrossRef]

34. Malloy, P.J.; Peng, L.; Wang, J.; Feldman, D. Interaction of the vitamin D receptor with a vitamin D response element in the Mullerian-inhibiting substance (MIS) promoter: Regulation of MIS expression by calcitriol in prostate cancer cells. Endocrinology 2009, 150, 1580-1587. [CrossRef]

35. Merhi, Z.; Doswell, A.; Krebs, K.; Cipolla, M. Vitamin D alters genes involved in follicular development and steroidogenesis in human cumulus granulosa cells. J. Clin. Endocrinol. Metab. 2014, 99, E1137-E1145. [CrossRef] [PubMed]

36. Irani, M.; Minkoff, H.; Seifer, D.B.; Merhi, Z. Vitamin D increases serum levels of the soluble receptor for advanced glycation end products in women with PCOS. J. Clin. Endocrinol. Metab. 2014, 99, E886-E890. [CrossRef]

37. Dennis, N.A.; Houghton, L.A.; Pankhurst, M.W.; Harper, M.J.; McLennan, I.S. Acute supplementation with high dose Vitamin D3 Increases serum anti-müllerian hormone in young women. Nutrients 2017, 9, 719. [CrossRef] [PubMed]

38. Dorgan, J.F.; Spittle, C.S.; Egleston, B.L.; Shaw, C.M.; Kahle, L.L.; Brinton, L.A. Assay reproducibility and within-person variation of Müllerian inhibiting substance. Fertil. Steril. 2010, 94, 301-304. [CrossRef]

39. Tal, R.; Seifer, D.B. Ovarian reserve testing: A user's guide. Am. J. Obstet. Gynecol. 2017, 217, 129-140. [CrossRef]

40. Barrea, L.; Muscogiuri, G.; Laudisio, D.; Pugliese, G.; de Alteriis, G.; Colao, A.; Savastano, S. Influence of the Mediterranean diet on 25- Hydroxyvitamin D levels in adults. Nutrients 2020, 12, 1439. [CrossRef] 